\title{
Bronchoscopy Practice in Turkey: A Questionnaire Study
}

\section{Ergun Tozkoparan¹, Benan Çağlayan², Levent Dalar³, Semra Bilaçeroğlu ${ }^{4}$, Ahmet Ilgazlı ${ }^{5}$, Interventional Pulmonology Study Group of Turkish Respiratory Society}

\author{
${ }^{1}$ Department of Pulmonary Medicine, Gülhane Military Medical Academy, Ankara, Turkey \\ ${ }^{2}$ Dr. Lütfi Kırdar Kartal Research and Trainig Hospital, Department of Pulmonary Medicine, İstanbul, Turkey \\ ${ }^{3}$ Department of Pulmonary Medicine, İstanbul Bilim University Faculty of Medicine, İstanbul, Turkey \\ ${ }^{4} \mathrm{Dr}$. Suat Seren Center of Chest Disease and Thoracic Surgery, Izmir, Turkey \\ ${ }^{5}$ Department of Pulmonary Medicine, Kocaeli University Faculty of Medicine, Izmit, Turkey
}

\begin{abstract}
Objective: As in the rest of the world, there are differences in attitudes and behaviours among physicians in bronchoscopy applications in our country as well. The aim of this study was to put forward the attitudes and behaviours of bronchoscopists using a questionnaire as the Working Group of Interventional Pulmonology of Turkish Respiratory Society.

Methods: The invitation letter prepared individuals for participation in the web-based questionnaire containing 23 questions on the implementation of diagnostic and interventional bronchoscopy; the link to the questionnaire was sent to the e-mail groups of pulmonologists, thoracic surgeons and anesthesia and intensive care specialists. The responses of the physicians participating to the questionnaire were evaluated. The results obtained from the questionnaire were evaluated generally and on the basis of medical branches by statistical analyses.

Results: The questionnaire was answered by a total of 146 specialists including 93 pulmonologists, 30 thoracic surgeons and 23 anesthesiologists. In total, $73 \%$ of the physicians responding to the questionnaire were working at the University or Training and Research Hospital and $47 \%$ of the respondents were conducting more than 100 bronchoscopies per year. Here, $36 \%$ of the respondents stated that in the bronchoscopies that they carried out, they did not sedate their patients. On the other hand, the most commonly preferred medication for sedation was midazolam (78\%). While $77 \%$ of the pulmonologists used nebulised lidocaine as a topical anesthetic, these rates were $12.5 \%$ and $8.3 \%$ respectively for thoracic surgeons and anaesthesiologists $(\mathrm{p}<0.001)$. Also, $38 \%$ of pulmonologists were conducting $23 \%$ of the diagnostic bronchoscopies under general anesthesia. During bronchoscopy, the pulse oximetry follow-up was found to be $97 \%$, and the supplemental oxygen administration was $87 \%$. Overall, $60 \%$ of the pulmonologists thought that at least 100 procedures must be performed under the supervision of a specialist during bronchoscopy training and $40 \%$ of them were of the opinion that the number of procedures undertaken must be at least 50 .
\end{abstract}

Conclusion: The data of the survey give information about the practice of bronchoscopy in Turkey. It is suggested that the repetition of such questionnaires by increasing the variety of questions and the number of participants will provide an important data source.

Keywords: Bronchoscopic procedures, guideline, questionnaire

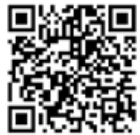

Received Date: 08.12 .2013

Accepted Date: 24.02.2014

Address for correspondence

Ergun Tozkoparan, Department of Pulmonary Medicine, Gülhane Military Medical Academy, Ankara, Turkey

E-mail: etozkoparan@gata.edu.tr

C Copyright 2014 Turkish Respiratory Society (TRS) Eurasian J Pulmonol 2014

DOI: $10.5152 /$ ejp.2014.93685

- Available online at www.eurasianjpulmonol.com

\section{INTRODUCTION}

As for many surgical and medical interventions, efforts to reach to the airways trace back to the period of Hippocrates (B.C. 460-370). Hippocrates suggested that placing a tube in the larynx of patients will prevent asphyxiation and facilitate respiration. The first bronchoscopy procedure in today's context was conducted by Dr Gustav Killian, who was a rhinolaryngologist in Germany in 1897 (1). Dr Killian removed a piece of pork bone that a patient had aspirated from the right main bronchus. After this first intervention by Dr Killian, fast progress was made technologically in bronchoscopy and the first rigid bronchoscope closest to those used today was developed by Dr Chevalier Jackson in the United States of America in 1904. Until the 1970s, rigid bronchoscopes had been used in the fields of chest diseases (CD) and thoracic surgery (TS). Dr Shigeto İkeda from Japan produced the prototype of flexible bronchoscopes for the first time with the company Olympus in 1968 (1). Since the beginning of the 1970s, after flexible bronchoscopes were supplied to the market, bronchoscopy has become an inevitable diagnosis and treatment method for pulmonologists and thoracic surgeons.

Although headway was made in the use of bronchoscopy as a diagnosis and treatment machine in a short time with the help of technology, there may be some differences in the bronchoscopy applications of the physicians. There are many points that cannot be compromised on, such as preparation before the procedure, anesthesia, sedation, and follow-up during and after the procedure. There 
Table 1. Questions included in the questionnaire

Question 1: Which institution do you work at?

Question 2: How long have you been working as a specialist physician?

Question 3: Which sections are included in your bronchoscopy unit? (recovery room, disinfection room, etc.)

Question 4: How are device cleaning and disinfection processes conducted in your bronchoscopy unit?

Question 5: How many bronchoscopy procedures do you perform in average in a year?

Question 6-13: While preparing the patient for bronchoscopy, which of the following examinations do you perform and how often? (a separate question for each examination)

Complete blood count, Coagulation Profile (aPTT, PTZ, INR), Routine biochemistry, markers of viral infectious diseases ( HBV, HCV, HIV etc.),Pulmonary

Function Tests, Thorax Tomography, Arterial Blood Gases analysis, ECG

* Question 14: Which of the following applications do you use for pre-bronchoscopy sedation and how often?

** Question 15: Can you get the support of an anesthesiologist for patients undergoing sedation?

Question 16: What do you prefer for atropine application?

* Question 17: Which of anesthesia methods do you prefer for routine diagnostic bronchoscopy and how often (local, general)?

* Question 18: Which of patient positions and application ways do you prefer for routine diagnostic bronchoscopy and how often?

* Question 19: Which of local anesthesia methods do you prefer for routine diagnostic bronchoscopy and how often?

* Question 20: How often do you perform following applications during bronchoscopy?

Pulse oximetry, ECG, Establishment of vascular access, Supplementary oxygen, Blood pressure follow-up

* Question 21: Which of the following interventional procedures do you perform and how often? How many cases do you examine per year?

Rigid bronchoscopy, Laser application, Electrocauterisation, Argon plasma coagulation (APC), Cryotherapy, Brachytherapy, Photodynamic therapy,

Balloon dilatation, Stent placement, Endobronchial ultrasonography (EBUS) (radial or convex probe), Alveoloscopy, Electromagnetic navigation (EMN),

Thermoplasty, Endobronchial valve application

*** Question 22: At least how many procedures must be performed under the supervision of a competent expert to have enough experience in routine diagnostic bronchoscopy?

Question 23: At least how many procedures must a trained bronchoscopist perform per year to maintain his/her experience and competence?

* Participants can choose more than one alternative for these questions.

** This question was not asked to anesthesiologists.

*** This question was asked only to pulmonologists.

have been various guidelines published for different bronchoscopy applications (interventional, diagnostic etc.) by various international associations and institutions (2-8). While these guidelines are actually "guiding", since randomised controlled clinical trials are generally inadequate, their evidence levels are relatively low compared to the guidelines in other medical practices. As some of the recommendations in many guidelines are based on specialist opinion, their evidence level was identified as $C$.

As in the rest of the world, there are differences in attitudes among physicians with regard to bronchoscopy applications in our country as well. The aim of this study was to put forward the attitudes and behaviours of bronchoscopists using a questionnaire as the Working Group of Interventional Pulmonology of Turkish Respiratory Society.

\section{METHODS}

The questionnaire, containing 23 questions prepared by the webbased program "Survey Monkey", was sent via e-mail to the target groups, including pulmonologists, thoracic surgeons and anesthesia specialists, twice in one month in June 2012. The specialist physicians from the related medical branches were requested to answer the survey, putting forward their attitudes and behaviours. Although questions about specialisation, duration working as a specialist physician in years and the quality of the institution they are working in (Univer- sity, research hospital, state hospital etc.) were posed, questions such as name, surname, title and name of the institution were not asked.

Apart from these first informative questions about the participant, questions such as those regarding the infrastructure of the bronchoscopy unit, anaesthetic methods used for routine diagnostic flexible bronchoscopy application, sedative agents used, examinations ordered before bronchoscopy and questions in different categories were used. Only the pulmonologists were asked to reply to questions about bronchoscopy. The answers of the questions were divided into subgroups according to the area of the specialist and are shown in percentages. Since some participants did not answer all of the questions, each question was analysed on the basis of the total number of replies. While a single alternative could be used in some questions, participants were allowed to mark more than one alternative in some questions. Approval of the Ethics Committee was not required for this study.

The survey questions are indicated in Table 1.

\section{Statistical Analysis}

The differences in the participant's replies according to the medical branches were analysed using the SPSS 17.0 software package and chi-square test. $\mathrm{P}<0.05$ value was accepted as significant. The answers found to be significant at the end of the statistical analysis are given in table 2 . 
Table 2. The questions with statistically different responses depending on the branches and the responses of CD, TS, A specialists

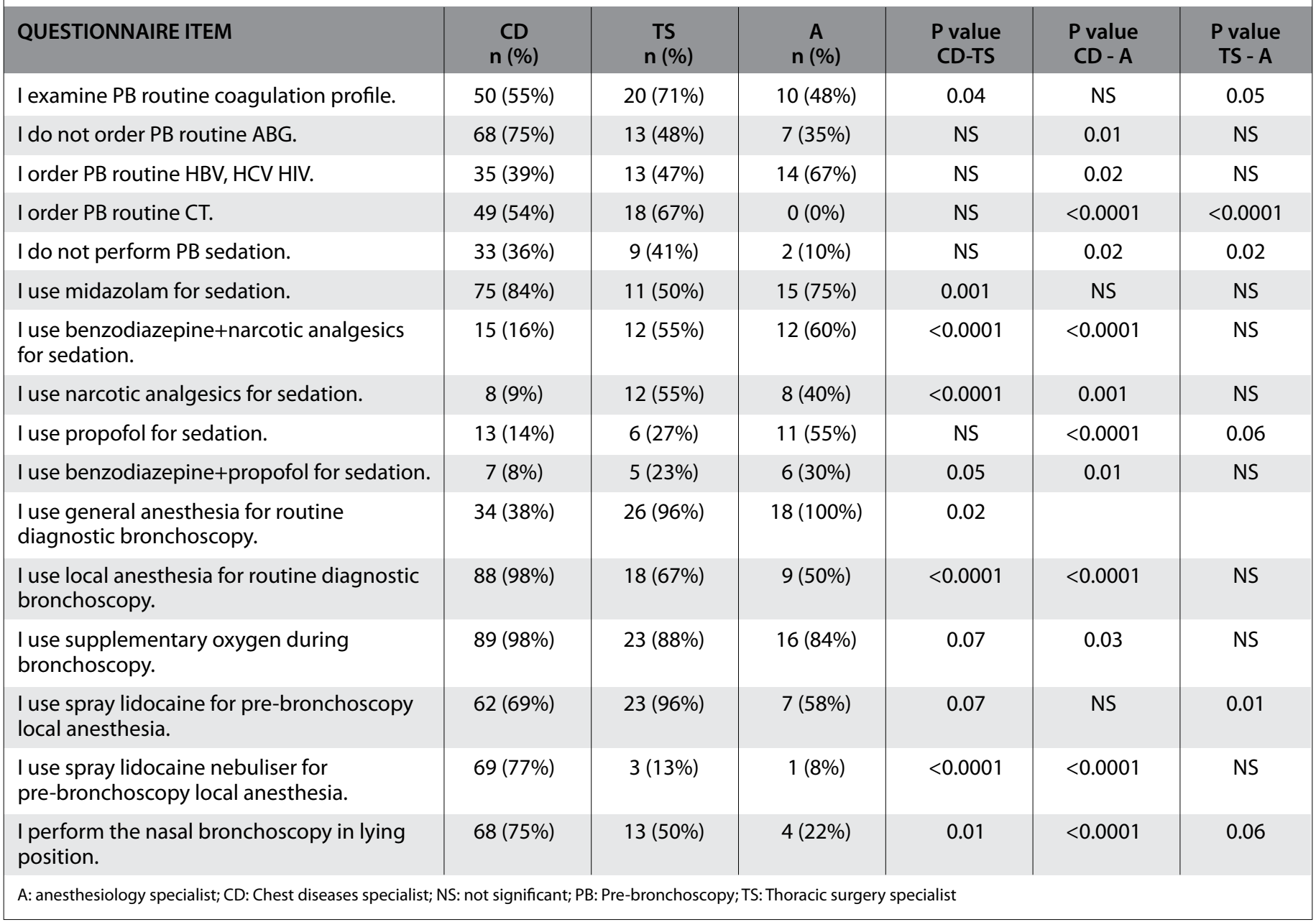

\section{RESULTS}

The questionnaire was answered by a total of 146 specialists, including 93 pulmonologists, 30 thoracic surgeons and 23 anesthesiologists. Overall, $36 \%$ of the participants were working in university hospitals, $14 \%$ of them in state hospitals and $14 \%$ of them in private hospitals. Also, $80 \%$ of the physicians had experience in their specialism for 6 years or longer.

\section{Bronchoscopy Unit}

Among the participant bronchoscopists, $47 \%$ of them were performing more than $100,10 \%$ of them between $80-100$ and $8 \%$ were performing between $40-60$ bronchoscopies per year. The remaining $28.7 \%$ of the physicians were performing less than 40 bronchoscopies per year.

Overall, $55 \%$ of the bronchoscopy units had a recovery room when the bronchoscopy units of all participants were taken into consideration; this percentage did not differ statistically between the branches ( $p>0.05$ ) (CD: $58 \%$, TS: 44\%, A: 55\%). All participants, including $\mathrm{CD}, \mathrm{TS}$ and $\mathrm{A}$ specialists, had disinfection and sterilisation rooms $(61 \%, 67 \%, 56 \%$ and $42 \%$ respectively). The cleaning and sterilisation of more than half of the bronchoscopies (57\%) were conducted manually and the others were cleaned and sterilised by automated machines. There were no differences observed among the medical branches $(p>0.05)$.

\section{Patient Preparation before Bronchoscopy}

When all of the participants were considered, before bronchoscopy, $37 \%$ of the physicians ordered blood chemistry (urea, creatinine, blood glucose, AST, ALT etc.), 57\% of them ordered a coagulation profile (aPTT, PT and INR), and $45 \%$ of them ordered viral infectious disease reagents (HBV, HCV, HIV). When an evaluation according to the medical branches is made, the ordering of a coagulation profile by CD specialists was significantly higher than that by TS specialists $(p=0.04)$. Moreover, while $68 \%$ of all the participants ordered a routine complete blood count before bronchoscopy, 14\% stated that if it was not required for the routine follow-up of the patient, they did not perform complete blood count. The rates of ordering complete blood count were $74 \%$ for CD specialists, $57 \%$ for TS specialists and $60 \%$ for A specialists (Figure 1).

Thoracic computed tomography (CT) was ordered by $48 \%$ of all the participants routinely before bronchoscopy and it was ordered in cases where transbronchial biopsy (TBB) or transbronchial needle aspiration (TBNA) would be conducted. On the other hand, $13 \%$ of all participants stated that they did not order thoracic CT before bronchoscopy. When evaluated according to the medical branches, while $54 \%$ of the CD specialists and $67 \%$ of the TS specialists stated that they wanted to see the BT of the patient before each bronchoscopy performed ( $p>0.05$ ), none of the A specialists found BT necessary $(p=0.0001$ for CD and TS specialists) (Figure 1). 


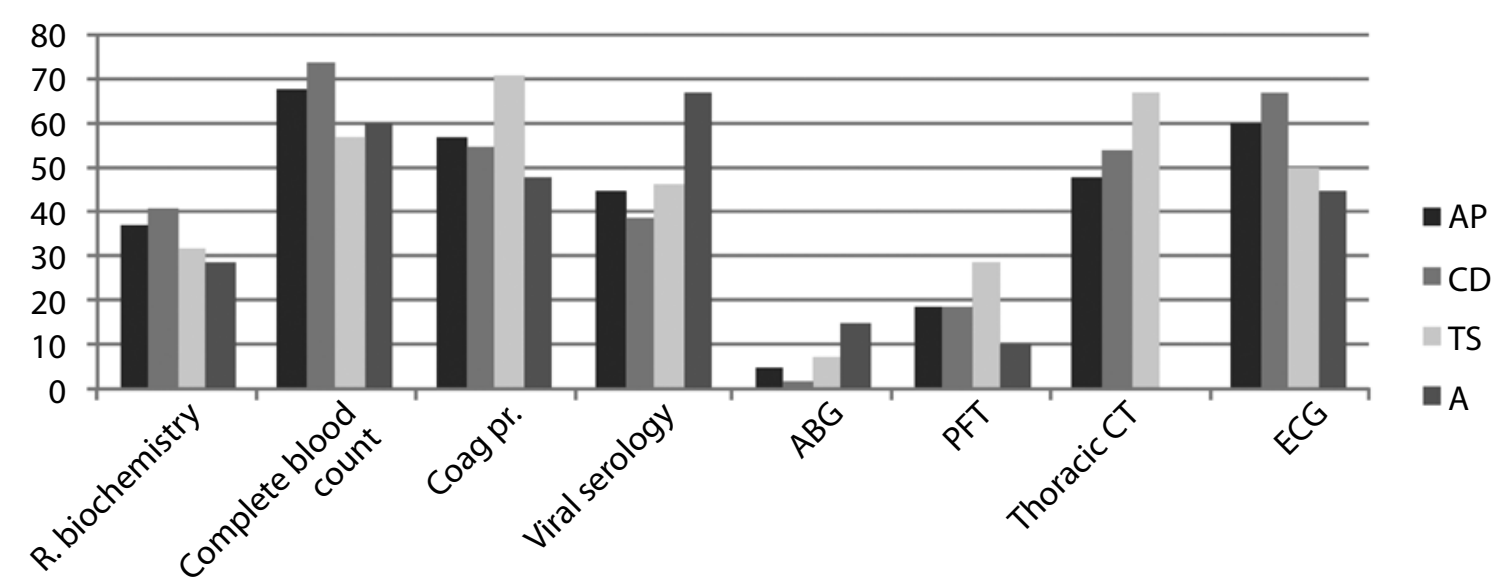

Figure 1. Pre-bronchoscopy tendency to order examinations for the physicians participating in the questionnaire (\%)

A: Anesthesiology specialists; ABG: Arterial blood gas; AP: All participants; CD: Chest diseases specialists; Coag. pr.: coagulation profile; ECG: Electrocardiogram; PFT: Pulmonary function test; R. Biochemistry: Routine biochemistry; Thorax CT: Computed tomography of the thorax; TS: Thoracic surgery specialists

While pulmonary function tests (PFT) are routinely ordered by $19 \%$ of the participants before bronchoscopy, $55 \%$ of the participants ordered them in case there were findings that made the physicians consider obstructive or restrictive pulmonary diseases; $25 \%$ of the participants did not order PFT before bronchoscopy. The ordering rate of pulmonary function tests (routinely or when necessary) was $75 \%$ for CD specialists, $78 \%$ for TS specialists and $43 \%$ for A specialists $(p>0.05)$.

While arterial blood gas ( $A B G$ ) analysis was ordered if low oxygen saturation $\left(\mathrm{SpO}_{2}\right)$ was detected by pulse oximetry or if pulmonary functions were impaired in $64 \%$ of the cases to whom bronchoscopy would be conducted, it was ordered by $5 \%$ of the participants routinely. On the other hand, $31 \%$ of the participants did not order ABG analysis, especially for bronchoscopy. While electrocardiography (ECG) evaluation was carried out routinely by $60 \%$ of the participants, $40 \%$ of them evaluated ECG when there was a suspicion of heart disease. Also, $67 \%$ of pulmonologists, $50 \%$ of TS specialists and $45 \%$ of A specialists ordered ECG routinely $(p>0.05)$ (Figure 1$)$.

\section{Premedication Sedation and Anesthesia}

Overall, $66 \%$ of the participants stated that they used general anesthesia and $85 \%$ of them stated that they used local anesthesia for routine diagnostic bronchoscopy. The physicians performing bronchoscopy under general anesthesia reported that they preferred to apply this method in $44 \%$ of all cases and those performing bronchoscopy under local anesthesia reported that they preferred to apply this method in $89 \%$ of cases. The rate of using local anesthesia was $98 \%$ for pulmonologists, $67 \%$ for TS specialists and $50 \%$ for A specialists. The difference between pulmonologists and TS specialists with regard to general anesthesia was significantly high in favour of pulmonologists $(p=0.02)$, and was significantly high in favour of TS specialists for local anesthesia. While $21 \%$ of all participants routinely applied atropine in bronchoscopy premedication, $37 \%$ of them applied it when bradycardia developed during the procedure. On the other hand, $42 \%$ of them stated that they did not apply atropine before or during bronchoscopy. These rates were $26 \%, 26 \%$ and $47 \%$, respectively, for pulmonologists, $11 \%, 44 \%$ and $44 \%$, respectively, for TS specialists and $9 \%, 76 \%$ and

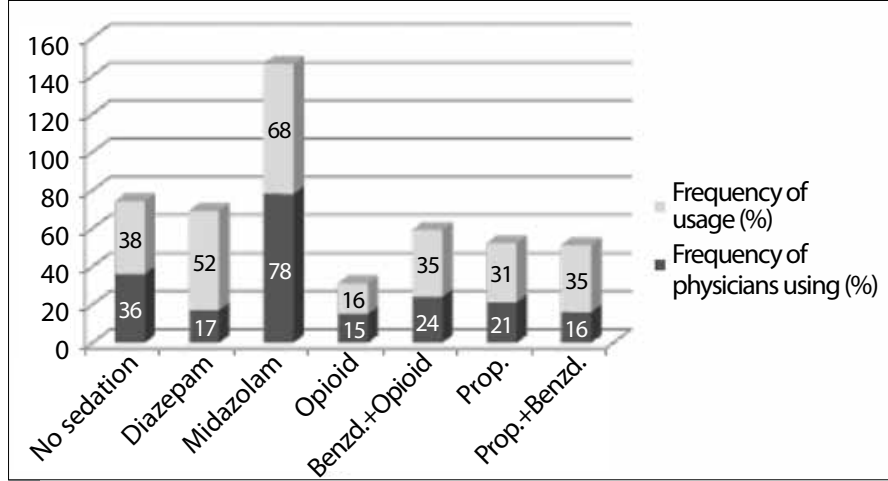

Figure 2. The tendencies of physicians participating in the questionnaire towards sedation and the frequency rates of the medications used for this aim

Benzd: benzodiazepine; No sedation: I do not perform sedation; Prop: propofol

$14 \%$, respectively, for A specialists. The most commonly preferred sedative agent was midazolam (78\% of all the physicians and $68 \%$ of their cases). Here, $36 \%$ of participants reported that they did not sedate patients in $38 \%$ of the bronchoscopies they conducted. The rates of the physicians reporting that they could perform bronchoscopy without sedating for CD, TS and A specialists were calculated as $36 \%, 41 \%$ and $10 \%$, respectively. Not performing sedation among CD, TS and A specialists was significantly different in favour of anesthesia specialists ( $p=0.02$ ) (Figure 2$)$. The rate of physicians reporting that they used propofol along with the other medications for sedation was calculated as $14 \%$ for CD specialists, $27 \%$ for TS specialists and $55 \%$ for A specialists $(p=0.0001)$. Midazolam was among the most preferred medications for $84 \%$ of CD specialists and $50 \%$ of TS specialists. $(p=0.001)$. The rates of agents used for sedation by the participator physicians and physician's percentage of the use of these medications for their patients are given in figure 2. Whether they took support from the anesthesia specialists for the sedated patients was asked to all participants except the anesthesia specialists. While the pulmonologists replied to this question as "yes", the rate of TS specialists' taking support from anesthesia was found to be $74 \%$. 
In bronchoscopy procedures conducted under local anesthesia, $67 \%$ of all participants preferred only topical lidocaine, $9 \%$ preferred nebulised lidocaine, $40 \%$ preferred both and $5 \%$ of them counted an application method except these as their preferences. These rates were found in CD specialists to be $69 \%, 77 \%, 39 \%$ and $2 \%$, respectively. The preference rate of nebulised lidocaine by pulmonologists was considerably higher compared to TS specialists.

\section{Bronchoscopy Procedure and Patient Follow-up}

When the replies of all participants were considered, it was noted that the frequencies of oral and nasal bronchoscopies in the lying position were $81 \%$ and $62 \%$, respectively, and the frequency of oral and nasal bronchoscopies in the sitting position were $17 \%$ and $21 \%$, respectively. Nasal bronchoscopy in the lying position was preferred by CD specialists compared to TS $(p=0.01)$ and A specialists $(p=0.0001)$ (Figure 3 ).

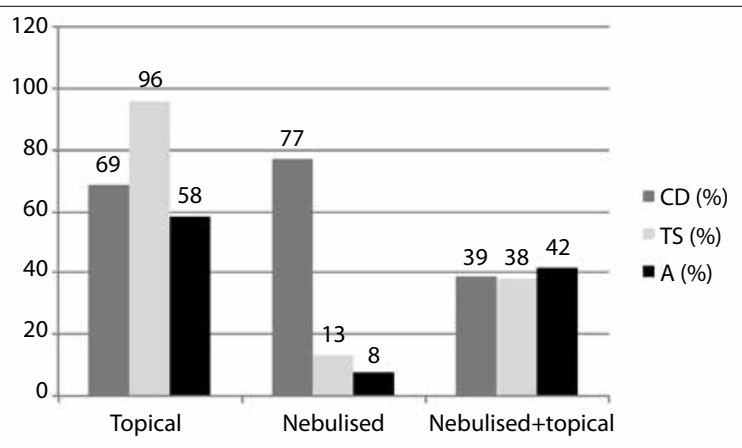

Figure 3. Preferences of the physicians participating in the questionnaire for the application of local anaesthetic agents according to their branches

CD: Chest diseases specialists; TS: Thoracic Surgery specialists; A: Anesthesiology specialists
The difference between thoracic surgeons and anesthesia specialists was found to be statistically insignificant $(p=0.06)$.

In following-up the patient during bronchoscopy, 97\% of all participants followed-up using pulse oximetry, 70\% of them used ECG monitoring, $87 \%$ used supplemental oxygen treatment and $71 \%$ of them followed-up blood pressure. These rates were $98 \%, 60 \%, 90 \%$ and $68 \%$, respectively, for the CD specialists and $90 \%, 84 \%, 80 \%$ and $71 \%$, respectively, for the TS specialists. While $\mathrm{SpO}_{2}$ and ECG follow-ups by pulse oximetry during bronchoscopy did not allow differentiation between the medical branches, supplemental oxygen treatment was considerably higher in $C D$ specialists compared to $A$ specialists $(p<0.05)$.

\section{Interventional Procedures and Education}

With regard to the interventional procedures, the participants were asked which and how many procedures they performed per year on average. Here, $29 \%$ of the pulmonologists performed rigid bronchoscopy and the average number of procedures they conducted was 35.5 per year. These numbers for pulmonologists were $66 \%$ and 80 procedure/year, respectively. The answers taken from the participants for other interventional procedures are found in Table 2.

The number of procedures that must be performed in diagnostic intervention in order to gaining sufficient experience under the supervision of a competent specialist was only asked to CD specialists. Here, $40 \%$ of the respondents stated that 50 procedures were sufficient, while $60 \%$ of them stated that it was necessary to perform at least 100 procedures. In response to the question of how many procedures a trained and experienced bronchoscopist has to conduct to maintain his experience and proficiency, $32 \%$ of the participants replied "below 50" and the remaining 68\% replied "50 and above".

Table 3. The number of bronchoscopists performing interventional bronchoscopy procedures and the average number of annual procedures

\begin{tabular}{|c|c|c|c|c|c|c|c|c|}
\hline \multirow[b]{2}{*}{$\begin{array}{l}\text { Interventional } \\
\text { procedure }\end{array}$} & \multicolumn{2}{|c|}{$\begin{array}{l}\text { All participants } \\
n=146\end{array}$} & \multicolumn{2}{|c|}{$\begin{array}{l}\text { Chest Diseases } \\
\quad n=93\end{array}$} & \multicolumn{2}{|c|}{$\begin{array}{l}\text { Thoracic Surgery } \\
\qquad n=30\end{array}$} & \multicolumn{2}{|c|}{$\begin{array}{l}\text { Anesthesia } \\
n=23\end{array}$} \\
\hline & $\begin{array}{c}\text { Number of } \\
\text { Bronchoscopists }\end{array}$ & $\begin{array}{c}\text { Number of } \\
\text { annual } \\
\text { procedures }\end{array}$ & $\begin{array}{c}\text { Number of } \\
\text { Bronchoscopists }\end{array}$ & $\begin{array}{c}\text { Number of } \\
\text { annual } \\
\text { procedures }\end{array}$ & $\begin{array}{c}\text { Number of } \\
\text { Bronchoscopists }\end{array}$ & $\begin{array}{c}\text { Number of } \\
\text { annual } \\
\text { procedures }\end{array}$ & $\begin{array}{c}\text { Number of } \\
\text { Bronchoscopists }\end{array}$ & $\begin{array}{l}\text { Number of } \\
\text { annual } \\
\text { procedures }\end{array}$ \\
\hline Rigid bronchoscopy & $54(37 \%)$ & 55.3 & $27(29 \%)$ & 35.5 & $20(67 \%)$ & 80.5 & $7(30 \%)$ & 52.5 \\
\hline Laser application & $10(7 \%)$ & 43.7 & $7(8 \%)$ & 52.4 & $6(20 \%)$ & 1.1 & 0 & 0 \\
\hline Electrocauterisation & $34(23 \%)$ & 11.6 & $16(17 \%)$ & 19.6 & $10(33 \%)$ & 8.1 & 0 & 0 \\
\hline APC & $19(13 \%)$ & 45.6 & $17(18 \%)$ & 49.8 & $1(3 \%)$ & 20 & 0 & 0 \\
\hline Cryotherapy & $14(10 \%)$ & 41.3 & $13(14 \%)$ & 43 & $1(3 \%)$ & 20 & 0 & 0 \\
\hline Brachytherapy & $1(0.6 \%)$ & 3 & $1(1 \%)$ & 3 & 0 & & 0 & 0 \\
\hline Balloon dilatation & $21(14 \%)$ & 15 & $14(15 \%)$ & 17.4 & $6(20 \%)$ & 5 & $1(4 \%)$ & 40 \\
\hline Stent & $29(20 \%)$ & 16.5 & $15(16 \%)$ & 24.8 & $12(40 \%)$ & 6.3 & $2(9 \%)$ & 32 \\
\hline EBUS & $17(12 \%)$ & 112.7 & $17(18 \%)$ & 112.7 & 0 & 0 & 0 & \\
\hline Alveoloscopy & $1(0.6 \%)$ & 7 & $1(1 \%)$ & 7 & 0 & 0 & 0 & \\
\hline EMN & $2(1.3 \%)$ & 152 & $2(2 \%)$ & 152 & 0 & 0 & 0 & \\
\hline Thermoplasty & 0 & 0 & 0 & & 0 & & 0 & \\
\hline Endobronchial valve & $8(5 \%)$ & 6.7 & $4(4 \%)$ & 3.2 & $2(7 \%)$ & 3 & $2(9 \%)$ & 9 \\
\hline
\end{tabular}




\section{DISCUSSION}

Diagnostic bronchoscopy, which is the most widely used method of bronchoscopy, is an intervention that is generally carried out by flexible bronchoscopy and characterised as being almost inevitable for $\mathrm{CD}$ specialists, differentiating them from the other internal medicine specialists. Whereas many international organisations, most notably the American Thoracic Society (ATS), European Respiratory Society (ERS), British Thoracic Society (BTS) and American College of Chest Physicians (ACCP), have tried to standardise bronchoscopy and other interventional pulmonology procedures to some extent, as bronchoscopy education, applications and experience are not uniform, both worldwide and in our country (2-8). For example, in a survey conducted by the ACCP, more than $50 \%$ of the participants stated that their training was not adequate for advanced diagnostic procedures such as TBNA (2).

By using this questionnaire, the aim was to take a snapshot of bronchoscopy applications in our country and to suggest general tendencies of the practitioners. When data obtained from the questions of the survey are evaluated, in our country, as in the rest of the world, there are differences between practitioners, either in the preparation stage or in the procedure; it was observed that these differences cause considerable differences in some areas among medical branches.

The most striking answer given to the question in the survey about structuring of bronchoscopy unit was that there was no recovery room in the bronchoscopy unit where $45 \%$ of all participants $(61 / 136)$ and $42 \%$ of CD specialists (38/90) worked. Moreover, manual cleaning of the machine in the bronchoscopy unit was carried out for $57 \%$ of all participants and $56 \%$ of CD specialists.

In some guidelines, automated machine use for bronchoscope disinfection is recommended in level of evidence $C$ (7). Nevertheless, it is pointed out that the disinfector and the bronchoscope must be compatible, otherwise the risk of contamination may increase (5). If the bronchoscope and the machine are not compatible, it will be more suitable to disinfect the equipment manually. While the compatibility of the disinfector and the machine was not questioned in this study, the rates obtained indicate that automated cleaning machines are still not widely used in our country.

Overall, $37 \%$ of the participants stated that they ordered routine biochemistry examinations, and $57 \%$ of them stated that they ordered routine coagulation profile examinations. In the published international guidelines, it is recommended in evidence level B not to order a complete blood count, blood biochemistry and coagulation parameters in case TBB is not performed and the patient does not have a known coagulopathy, chronic liver or kidney disease $(3,5,7)$. In the British Thoracic Society guidelines, if TBB is to be performed, it is recommended in evidence level $C$ to order the above-counted biochemical parameters before procedures, even if the patient does not have a known risk factor (3). In the questionnaire, separate questions were asked about routine blood biochemistry and coagulation parameters. In the questionnaire, the level of physicians who stated that they wanted to see the coagulation parameters for the patients to be performed for TBB and TBNA was 21\%. However, it was noted that the coagulation profile was ordered more by TS specialists than by $C D$ specialists. In answer to the question about indicators of viral infectious diseases (HBV, HCV, HIV), whereas $45 \%$ of the participants stated that they ordered these examinations routinely before procedure, the distribution of "Yes" replies according to the area of specialisation was $39 \%$ for CD specialists, $46 \%$ for TS specialists and $67 \%$ for $A$ specialists. The reason why anesthesiologists more frequently order infection indicators compared to other medical branches is thought to be the habit of ordering these examinations in all patients to be operated upon. The same reason may be valid in the more frequent ordering of the coagulation profile by TS specialists. In the existing guidelines, there are no recommendations about performing these examinations before procedures for infection control. Blood examination, either for coagulation profile or viral infectious diseases, is often ordered unnecessarily. It is understood that pulmonary function tests are ordered for every case by $19 \%$ of the participants. In the existing guidelines, it is recommended not to order PFT in evidence level $C$ as long as there is no clinical finding that make the physician consider an impaired respiratory function. Routine evaluation of ECG is not recommended in the guidelines for cases without a known heart disease. On the other hand, arterial blood gas is recommended in cases with advanced airway obstruction (FEV1/FVC $<50 \%$ or FEV $1<1 \mathrm{~L})$ or oxygen desaturation $\left(\mathrm{SpO}_{2}<93 \%\right)$; apart from this, routine examinations are not recommended $(3,5,7)$. Although the respondents of the survey generally replied appropriately to the guidelines, they had a different attitude by ordering ECG routinely at a rate of $60 \%$ before bronchoscopy. We are of the opinion that similar thoughts play a role here. As can be understood from the study, excessive amounts of examinations are ordered in our country. The reasons for this condition should be investigated and in case there is a lack of education it should be eliminated. It is possible that the physicians may order an excessive amount of examinations in case of possible complications, making themselves feel secure with regard to the law, and to prevent violence from the patient's relatives. As an anesthesia method, $66 \%$ of all the participants stated that they use general anesthesia and $85 \%$ of them stated that they use local anesthesia during bronchoscopy. It was noted that pulmonologists prefer local anesthesia more than TS and A specialists $(p<0.05)$. The high rate of general anesthesia usage among pulmonologists and A specialists can be explained by pulmonologists' performing bronchoscopy before operation under general anesthesia.

When the medicine spectrum that the participants use as a sedative agent during procedures is considered, it can be seen that midazolam was the leading medication, with a rate of $78 \%$. Although there is no definite recommendation in the guidelines about which sedative agent should be preferred, it is suggested that midazolam is the most commonly used medication in the studies (8). In their survey, Honeybourne et al. (9) reported that 112 of the 218 bronchoscopy units which used intravenous sedation used midazolam; opioids were used in 68 units and midazolam with opioids was used in 41 units. While in all guidelines it is recommended to sedate if there is no contraindication, it is remarkable that $36 \%$ of bronchoscopists participating in our study did not sedate in $38 \%$ of cases. It is indicated in the studies that sedation increases the tolerance of the patient and patient/physician comfort. Moreover, when it is necessary to repeat a procedure for the second time, in those cases that were sedated, it was found that the possibility of the patient accepting the procedure was higher $(2,8)$.

The most secure agent as a topical anaesthetic recommended in all guidelines is lidocaine $(3,5,7,8)$. Almost $97 \%$ of the participants of 
the survey stated that they use lidocaine in topical anesthesia. However, lidocaine application methods vary (spray, gel, nebulisation). There is no clear recommendation in the guidelines. Among the local anesthetic agents used during bronchoscopy procedures, the efficiency of cocaine (4\%), benzococaine (20\%), tetracaine (1\%) and lidocaine (1-10\%) are the same. Nevertheless, cocaine has a risk of misuse and adverse cardiovascular effects. The methemoglobinemia risk of benzocaine and tetracaine is higher than that of lidocaine, meaning that they must be used cautiously. Lidocaine is less toxic and is effective for a shorter time period. These reasons led to lidocaine being the preferred agent. Although in the first studies about lidocaine nebulisation it was reported that this method increased the patient's comfort, it was suggested in more recent randomised placebo-controlled studies that nebulised lidocaine did not increase patient comfort (8).

When the preferences of patient positions during procedure are considered, $75 \%$ of the CD specialists reported that they used nasal bronchoscopy in the lying position and $80 \%$ of them reported that they used oral bronchoscopy in the lying position. When anesthesiologists and TS specialists are considered, their preferences for oral bronchoscopy in lying position were $83 \%$ and $88 \%$, respectively. On the other hand, nasal bronchoscopy in the lying position was not preferred among anesthesiologists and CD specialists $(22 \%$ and $50 \%$, respectively). In fact, bronchoscopy can be performed nasally or orally. The advantages and disadvantages of the two methods in comparison to one another could not be indicated. However, more than half of the bronchoscopists prefer nasal bronchoscopy. Annakkaya et al. (10), reported in their prospective study that during the bronchoscopy performed in lying and sitting positions, there were no significant differences between systolic and diastolic blood pressures and average oxygen saturations. In this study, in the evaluations of patients after bronchoscopy, the tolerance to the procedure, difficulty of the procedure, cough and zonesthesia scores were found to be similar. In entering from the nasal airways, the nasal mucosa may be damaged and pain may occur after the procedure. If there is impairment in the nasal passage anatomy, the oral passage should be used without forcing the nasal passage. A mouthpiece must be used to enter from the oral airways. Otherwise, the patient may bite and damage the bronchoscope. While the majority of bronchoscopists prefer performing the procedure from behind the patient's head, some prefer to be in front of the patient in a sitting position. It is generally recommended that the bronchoscopist should use the method that he is most used to.

The questions about patient monitoring during bronchoscopy were answered by the participants, and were consistent with the guidelines. Overall, $97 \%$ of participants stated that they used pulse oximetry and $95 \%$ stated that they established vascular access routinely. In all guidelines, the routine establishment of vascular access and pulse oximetry are recommended. In the guidelines of the British Thoracic Society, although there are no recommendations about blood pressure follow-up, ECG is suggested for patients with severe heart disease and for hypoxic patients who do not respond to supplementary oxygen (3). In two other guidelines (Australia-New Zealand Thoracic Society and Israel Lung Association), ECG and blood pressure follow-up are recommended for all patients routinely $(5,7)$. Smyth et al. (11) reported that pulse oximetry follow-up during bronchoscopy was performed in their study in $99 \%$ of cases and ECG monitoring was carried out in $22 \%$ of cases routinely in the United Kingdom. Considering all of the physicians in our study, $90 \%$ of the participants stated that they performed ECG in 77\% of the bronchoscopy procedures; similarly, $92 \%$ reported that they followed-up blood pressure during the procedure in $95 \%$ of cases. No statistical difference was found between the medical branches in terms of electrocardiography and pulse oximetry follow-up.

Considering evaluation of the responses in terms of interventional bronchology, it can be seen that all procedures except thermoplasty are performed by some of the participants. Since it is not expected for all interventional procedures to be performed in all health centres and by all bronchoscopists, the use of all interventions at least in one centre in our country is a satisfying result.

Since we believe that the physicians from other branches (thoracic surgery, anesthesia, intensive care, pediatrics) can only perform bronchoscopy if they receive adequate education from CD specialists, we only wanted to ask questions about bronchoscopy education to $C D$ specialists. The number of procedures necessary for obtaining enough experience under the supervision of an expert was stated to be 50 by $40 \%, 100$ by $40 \%$ and 150 or more by the rest of the participants. This number must be at least 100 according to the guidelines of the American College of Chest Physicians (2). The Training Programme on Specialisation in Chest Diseases of Turkish Chest Diseases Competency Board (TCDCB) recommends at least 75 flexible and 20 rigid bronchoscopy applications during training. It is not known how many of the institutions providing specialisation training reach this number. Moreover, the Competency Board suggests the minimum number of EBUS to be 10 and the minimum number of interventional bronchoscopy to be 10 (2). Considering all of the institutions offering training programmes, it is known that EBUS and interventional bronchoscopy are not available in all units. It is obvious that educational institutions should study in cooperation for this issue. An educated bronchoscopist is recommended to perform at least 25 procedures per year to maintain competence. Of the participants, the necessary number of bronchoscopy procedures was stated to be 10 by $8 \%, 25$ by $23 \%, 50$ by $40 \%, 75$ by $8 \%$ and 100 by $21 \%$ of the physicians.

In conclusion, the data obtained from this questionnaire provide information about the experience of bronchoscopy in our country. In fact, with the results of this study, we have recognised that many different bronchoscopy procedures are available in our country, as in the rest of the world. Interestingly, although there are published guidelines for diagnostic bronchoscopy, it has been detected in questionnaire studies conducted in countries like the USA and England that a great majority of bronchoscopists do not follow these guidelines. Some of the differences in bronchoscopy (for example, patient position) caused by performers are not as important for patient safety and efficiency of the procedure. However, more standardised approaches should be suggested for issues such as sedation, topical anesthesia applications, patient safety during the procedure and preparation before the procedure. This would be possible with further studies about the efficiency and safety of bronchoscopy. In our country, we can design our guidelines in line with the increased number and quality of these studies and have standardised approaches for bronchoscopy. 
Informed Consent: N/A.

Peer-review: Externally peer-reviewed.

Author Contributions: Concept - B.C., E.T., L.D., S.B., A.I.; Design - E.T., B.C.; Supervision - B.C.; Resource - E.T., B.C.; Analysis\&/or Interpretation - E.T., B.C.; Literature Search - E.T.; Writing - E.T., B.C.; Critical Reviews - L.D.

Acknowledgements: Authors thank to the all respoders of the questionaire for their valued contributions.

Conflict of Interest: All of the authors declared no conflict of interest.

Financial Disclosure: The authors declared that this study has received no financial support

\section{REFERENCES}

1. Becker HD. A Short History of Bronchoscopy. In: Ernest A, editor. Introduction to Bronchoscopy. 2nd ed. Newyork: Cambridge Universty Press; 2009.

2. Ernst A, Silvestri GA, Johnstone D, American College of Chest Physicians. Interventional Pulmonary Procedures: Guidelines from the American College of Chest Physicians. Chest 2003; 123: 1693-717.

3. British Thoracic Society Bronchoscopy Guidelines Committee, a Subcommittee of Standards of Care Committee of British Thoracic Society. British Thoracic Society guidelines on diagnostic fexible bronchoscopy. Thorax 2001; 56: i1-21.

4. Du Rand IA, Rintoul RC, Barber PV, Shah PL, Goldring J, Singh S et al. on behalf of the British Thoracic Society Interventional Bronchoscopy Guideline Group. British Thoracic Society guideline for advanced diagnostic and therapeutic flexible bronchoscopy in adults. Thorax 2011; 66: iii1-21.
5. Wood-Baker R, Burdon J, Mcgregor A, Robinson P, Seal P, Thoracic Society of Australia and New Zealand. Fibre-optic bronchoscopy in adults: a position paper of The Thoracic Society of Australia and New Zealand. Intern Med J 2001; 31: 479-87.

6. Bolliger CT, Mathur PN. Beamis JF, Becker HD, Cavaliere S, Colt, et al. ERS/ ATS statement on interventional pulmonology. Eur Respir J 2002; 19: 356-73.

7. Shulimzon TR, Israel Lung Association Task Force. Flexible Bronchoscopy in israel 2010: evidence-Based clinical Practice guidelines for the adult patient: a concise summary of the recommendations of the recommendations of the Israel Lung Association Task Force. Isr Med Assoc J 2010; 12: 69-73.

8. Wahidi MM, Prasoon J, Mackensen, Jantz M, Lee P, Burkhard G, et al. Flexible Bronchoscopy in Adult Patients Anesthesia, Analgesia, and Sedation During Consensus Statement on the Use of Topical American College of Chest Physicians. 2011; 140: 1342-50.

9. Honeybourne D, Neumann CS. An audit of bronchoscopy practice in the United Kingdom: a survey of adherence to national guidelines. Thorax 1997; 52: 709-13.

10. Annakkaya AN, Bedirhan I, Tozkoparan E, Özkan M, Yüksekol I, Bilgiç $H_{\text {, }}$ et al. Patient position during fiberoptic bronchoscopy: sitting or supine? Respiratory Diseases 2003; 14: 266-74.

11. Smyth CM, Stead RJ. Survey of flexible fibreoptic bronchoscopy in the United Kingdom. Eur Respir J 2002; 19: 458-63.

12. Kocabaş A, İtil O, Durak H.I, Törün SE, Taşkıran H.C. Qualifications board of the turkish chest diseases, pulmonary disease specialist training program. İstanbul: Probiz, 2010. 